\title{
Attempt of an Axiomatic Foundation of Quantum Mechanics and More General Theories. $\mathrm{IV}^{\star}$
}

\author{
GÜNTER DÄHN \\ Institut für theoretische Physik (I), der Universität Marburg
}

Received March 21, 1968

\begin{abstract}
This contribution continues the series of papers on the same subject which has been treated by LUDwIG in [1-3]. Using the system of axioms as given in [3], we shall succeed in constructing an orthomodular lattice of linear operators on the real vector space generated by the physical decision effects. There results an isomorphism between the orthomodular lattice of all physical decision effects and the lattice to be constructed.
\end{abstract}

\section{Preliminaries}

As shown by Foulis ([7-11]), any orthomodular lattice can be coordinatized by a Baer-*-semigroup (i.e. a $*$-semigroup where the annihilator of each element is a principal left (right) ideal generated by a selfadjoint idempotent). At this point the theory developed becomes relevant for physics: On the one hand, PooL $[17,18]^{1}$ has given the concept of a Baer-*-semigroup a direct physical meaning by including the ideal measuring process in an axiomatic lattice approach to quantum theories. On the other hand, the multiplicative semigroup in the ring of all bounded linear operators on Hilbert-space is such a semigroup. But looking for structures for physics like Hilbert-space, we notice that the mathematical situation is still more complicated: As it has generally turned out by LUDWIG's system of axioms, the lattice to be co-ordinatized is embedded in a topological vector space. MrLes [12] has given the problem the most general form: Let $\mathscr{A}$ be a $B^{*}$-algebra. Its selfadjoint elements form a real partially ordered vector space $H(\mathscr{A})$ with a positive cone of elements $x^{*} x$ for all $x \in \mathscr{A}$. Required is the knowledge in how far $\mathscr{A}$ is already determined by $H(\mathscr{A})$. Ideally we should have to find the class of all $B^{*}$-algebras $\mathscr{A}$ for which for a given real partially

* This paper is a modified version of the author's thesis ,Zur Koordinatisierung des orthomodularen Verbandes physikalischer Entscheidungseffekte", written at Marburg University under the direction of Prof. G. LuDwig. It was supported in part by the Deutsche Forschungsgemeinschaft.

1 I am indebted to Prof. R. HAAG for having directed my attention to these papers. 
ordered vector space $H$ the space $H(\mathscr{A})$ is isomorphic to $H$ with respect to order and vector space structure. So general the problem has not yet been solved up to now.

In this paper we shall try to tackle the problem in question by imposing finite dimension on $H$. But this will lead us only to the partial result that the orthomodular lattice $G$ of the physical decision effects in $H$ can be isomorphically mapped on an orthomodular lattice in the algebra $\mathscr{B}(H)$. It could not yet be decided whether this algebra is a $B^{*}$-algebra.

Since here we concern ourselves only with purely mathematical investigations, we omit any physical motivation of the concepts and axioms established by LUDWIG.

Detailed physical discussions can be found in [4-6]. Yet to make this exposition more readable, we will briefly quote the most important definitions and the system of axioms given by LuDwig. Although it is possible to formulate them mathematically more weakly, we do not so because, as shown in [1-3], the here given formulation can be derived from LuDwig's original one. Our formulation will be most adapt to our purposes.

As a conceptual frame we have a dual pair $\left(B, B^{\prime}\right)$ of real topological vector spaces which we shall, throughout this paper, suppose to be finite-dimensional. Henceforth this supposition shall tacitly be included in all those theorems and statements which only hold by argumentation using finite dimension of $B$ (and hence of $B^{\prime}$ ).

$B$ is spanned by the closed convex hull $K$ of the set $K$ of all physical ensembles $V$. The elements of $K$ are denoted by $V . B^{\prime}$ is spanned by the weak closure $L$ of the set $L$ of all physical effects $F$. The elements of $L$ are denoted by $F$. It is mathematically necessary to introduce the weakly closed convex hull $\hat{L}$ of $L$. The elements of $\hat{L}$, too, are denoted by $F$, since they can be interpreted physically ([4-6]). The topologies adverted to will be defined after axiom 1 . The sets $K$ and $\hat{L}$ are put in duality by that

Axiom 1. There exists a mapping $\mu$ on $K \times \hat{L}, \mu: K \times \hat{L} \rightarrow \boldsymbol{R}_{+}$so

$(\alpha) o \leqq \mu(V, F) \leqq 1$ for all $(V, F) \in K \times \hat{L}$

( $\beta$ ) for all $V_{1}, V_{2} \in K: \mu\left(V_{1}, F\right)=\mu\left(V_{2}, F\right)$ for all $F \in \hat{L}$ implies $V_{1}=V_{2}$

$(\gamma)$ for all $F_{1}, F_{2} \in \hat{L}: \mu\left(V, F_{1}\right)=\mu\left(V, F_{2}\right)$ for all $V \in K$ implies $F_{1}=F_{2}$

( $\delta$ ) there exists $F_{o} \in \hat{L}$ (denoted by $O$ ) so that $\mu(V, O)=o$ for all $V \in K$

(ع) for each $V \in K$ there exists $F \in \hat{L}$ so that $\mu(V, F)=1$. 
$\mu$ can be so extended to $B \times B^{\prime}$ that it coincides with the canonical bilinear functional $\langle\cdot, \cdot\rangle$ over $B \times B^{\prime}$. In $B$ we define a norm by

$$
\|X\|:=\sup \{|\mu(X, F)| \mid F \in L\} \text { for all } X \in B .
$$

Thus any $V \in K$ satisfies $\|V\|=1$. With respect to this norm $B$ becomes a Banach-space and the closures in $B$ are taken in the pertaining norm topology. The space of all continuous linear functionals over $B$ is $B^{\prime}$. It is also a Banach-space by

$$
\|Y\|:=\sup \{|\mu(X, Y)| \mid X \in B,\|X\|=1\} \text { for all } Y \in B^{\prime} .
$$

Thus any $F \in L$ (and hence any $F \in \hat{L}$ ) satisfies $\|F\| \leqq 1$. Besides, we can define the so-called weak topology in $B^{\prime}$. The sets $L$ and $\hat{L}$ are defined with respect to this topology. But because of our dimension hypothesis we need not distinguish between norm and weak topology in $B^{\prime}$. By the definition:

"for all $Y_{1}, Y_{2} \in B^{\prime}: Y_{1} \leqq Y_{2}$ iff $\mu\left(V, Y_{1}\right) \leqq \mu\left(V, Y_{2}\right)$ for all $V \in K$ " $\mathrm{B}^{\prime}$ becomes a partially ordered, real Banach-space.

For all $l \leqq \hat{L}$ we define

$$
\begin{aligned}
& K_{o}(l):=\{V \mid V \in K, \mu(V, F)=o \text { for all } F \in l\} \\
& K_{1}(l):=\{V \mid V \in K, \mu(V, F)=1 \text { for all } F \in l\}
\end{aligned}
$$

For all $k \leqq K$ we define dually

$$
\begin{aligned}
& L_{o}(k):=\{F \mid F \in L, \mu(V, F)=o \text { for all } V \in k\} \\
& \hat{L}_{o}(k):=\{F \mid F \in \hat{L}, \mu(V, F)=o \text { for all } V \in k\} \\
& L_{1}(k):=\{F \mid F \in L, \mu(V, F)=1 \text { for all } V \in k\} \\
& \hat{L}_{1}(k):=\{F \mid F \in \hat{L}, \mu(V, F)=1 \text { for all } V \in k\}
\end{aligned}
$$

Let us consider for any $l_{o} \leqq \hat{L}$ the greatest $l_{g} \leqq \hat{L}$ so that $K_{o}\left(l_{o}\right)=K_{o}\left(l_{g}\right)$. There obviously holds $l_{g}=\cup\left\{l \mid K_{o}\left(l_{o}\right)=K_{o}(l)\right\}$. In [1-3] it was shown that any $l_{g}$ can be represented as $l_{g}=\hat{L}_{o} K_{o}(l)$ with $K_{o}(l)=K_{o}\left(l_{g}\right)$. By

Axiom 2a. For every pair $F_{1}, F_{2} \in \hat{L}$ there exists $F_{3} \in \hat{L}$ so that $F_{1} \leqq F_{3}, F_{2} \leqq F_{3}$ and $K_{o}\left(F_{3}\right) \supseteqq K_{o}\left(F_{1}\right) \cap K_{o}\left(F_{2}\right)$.

Any $l_{g}$ turns out to be an ascending directed set possessing a greatest element $E_{l_{g}}$. This is defined by

$$
\mu\left(V, E_{l_{g}}\right):=\sup \left\{\mu(V, F) \mid F \in l_{g}\right\} \quad \text { for all } \quad V \in K
$$

and satisfies $\left\|E_{l_{g}}\right\|=1$.

It would have been sufficient (see $[1-3]$ ) to formulate the axioms 1 and 2 a for $L$. Nevertheless we should have obtained analogous results, in particular: for each $k \leqq K, L_{o}(k)$ has the same $E$ as $\hat{L}_{o}(k)$. The set of all $E$ is denoted by $G$, its elements are called decision effects. 
Let us define the sets

$$
\begin{aligned}
U: & =\left\{L_{o}(k) \mid k \leqq K\right\}, \hat{U}:=\left\{\hat{L}_{o}(k) \mid k \leqq K\right\}, W:=\left\{K_{o}(l) \mid l \leqq L\right\} \\
\hat{W}: & =\left\{K_{o}(l) \mid l \leqq \hat{L}\right\} .
\end{aligned}
$$

As verified in [1-3], $U, \hat{U}, W$, and $\hat{W}$ are complete lattices with zero and unit elements. Between $U$ and $\hat{U}$ there exists a lattice isomorphism, whereas $W$ is equal to $\hat{W}$. Between $U$ and $W$ there exists a dual lattice isomorphism. The zero elements in $U,(\hat{U})$ and $W$ are $\{0\}$ and $\emptyset$, respectively. The units are $L,(\hat{L})$ and $K$, respectively. $G$ is lattice-isomorphic to $U$ and $\hat{U}$; thus dually isomorphic to $W$. The unit element 1 in $G$ is given by: $\mu(V, \mathbf{1})=1$ for all $V \in K$. By $\mu\left(V, E^{\prime}\right)$ $=1-\mu(V, E)$ for all $V \in K, G$ becomes orthocomplemented and so do $U, \hat{U}$ and $W$. Furthermore, the lattices are orthomodular or, equivalently, segment-orthocomplemented (see [1-3]). That means: for every segment $G(O, E) \leqq G$ and each $E_{1} \in G(O, E)$ there exists a unique element $E_{2} \in G(O, E)$ so that $E=E_{1} \stackrel{\perp}{\vee} E_{2}$, where $E_{1} \stackrel{\frac{1}{V}}{V} E_{2}$ is the abbreviation for $E_{1} \vee E_{2}$ and $E_{1} \perp E_{2}$, i.e. $E_{1} \leqq E_{2}^{\prime} . E_{2}$ is given by $E_{2}=E \wedge E_{1}^{\prime}$. Besides, in $G$ we have for every finite set of orthogonal elements: $\underset{i=1}{\stackrel{n}{V}} E_{i}=\sum_{i=1}^{n}$ $E_{i}, n$ any finite integer, $\Sigma$ denoting addition in $B^{\prime}$. All these facts can be found in [3], for instance. $\wedge$ and $\vee$ denote lattice-theoretical intersection and union, respectively. The lattice-theoretical intersection coincides with the set-theoretical one; but in general, the lattice-theoretical union differs from the set-theoretical one: in $\hat{U}$ and $W$, for instance, it is given by forming the least extremal set containing the set-theoretical union of all sets to be united lattice-theoretically.

To formulate axiom $2 \mathrm{~b}$ we first introduce the set

$$
\left\{Y \mid Y \in B^{\prime}, Y=\lambda F, \lambda \in \boldsymbol{R}_{+}, F \in \hat{L}, \mu(V, Y) \leqq 1 \text { for all } V \in K\right\} \text {. }
$$

This set is obviously convex, hence so is its (weak) closure $\hat{\bar{L}}$.

Axiom 2b. For all $F \in \widehat{\hat{L}}$ and all $E \in G$ :

$$
K_{o}(F) \supseteqq K_{o}(E) \text { implies } F \leqq E \text {. }
$$

This axiom is important concerning the structure of the positive cone in $B^{\prime}$ (see [3]). In [3] LuDwIG considers the cones

$$
\begin{aligned}
\mathscr{P}:= & \left\{Y \mid Y \in B^{\prime}, Y \geqq O\right\}, \mathscr{P}_{+}:=\left\{Y \mid Y \in B^{\prime}, \mu(V, Y) \leqq 1\right. \\
& \quad \text { for all } V \in K\} \\
\mathscr{Q}:= & \{X \mid X \in B, \mu(X, Y) \geqq o \text { for all } Y \in \mathscr{P}\} .
\end{aligned}
$$

Theorem 1 collects some properties of these cones, which were derived in [3]: 
Theorem 1. $\mathscr{P}=\bigcup_{\lambda \in R_{+}} \lambda \cdot \hat{\hat{L}}, \mathscr{P}_{+}=1-\mathscr{P}$

(ii) $\hat{L}=\hat{L}, \hat{L}=\mathscr{P} \cap \mathscr{P}_{+}$

(iii) $\mathscr{Q}=\bigcup_{\lambda \in R_{+}} \lambda K$

(iv) $B^{\prime}=\mathscr{P}-\mathscr{P}, B=\mathscr{Q}-\mathscr{Q}$.

Let us denote by $C(V)$ the smallest, extremal set of $K$ containing $V$ (see def. 2). As the last axiom for our exposition we formulate:

Axiom 3. For every pair $V_{1}, V_{2} \in K$ :

$$
\hat{L}_{o}\left(V_{1}\right)=\hat{L}_{o}\left(V_{2}\right) \text { implies } C\left(V_{1}\right)=C\left(V_{2}\right) \text {. }
$$

The converse implication holds always.

It is now appropriate to give a mathematical outline of what we finally bear in mind: For any $E \in G$ we shall show that the pair $\left(K_{1}(E), \hat{L}_{E}\right)$ with $\hat{L}_{E}:=\{F \mid F \in \hat{L}, F \leqq E\}$ is as dual as $(K, \hat{L})$. Then we consider their corresponding vector spaces and define a projector $\boldsymbol{T}_{E}$ of $B^{\prime}$ onto $B(E)^{\prime}$ determined up to an isomorphism by $\hat{L}_{E}$. The set $\mathscr{T}(G)$ of all $\boldsymbol{T}_{E}$ forms an orthomodular lattice isomorphic to $G$. By $\mathscr{T}(G)$ we generate a subalgebra of $\mathscr{B}\left(B^{\prime}\right)\left(\mathscr{B}\left(B^{\prime}\right)\right.$ being the set of all (bounded) operators linear on $\left.B^{\prime}\right)$. To verify duality of $\left(K_{1}(E), \hat{L}_{E}\right)$ we need some results on extremal sets and facets. To them the next section is dedicated.

\section{Extremal Sets and Facets}

For the sake of convenience let us quote two definitions given by DAY in [13] chap. V.

Definition 1. (i) For any convex subset $A$ of a vector space: $p \in A$ is a passing point iff $p$ belongs to an open segment $S \leqq A$.

(ii) $e \in A$ is an extreme point of $A$ iff it is not a passing point.

Definition 2. For any closed convex subset $K$ of a topological (locally convex) vector space: $A \leqq K$ is an extremal set of $K$ iff

(i) $A \neq \emptyset, A$ convex and closed

(ii) every open line segment $S \subset K$ with $S \cap A \neq \emptyset$ satisfies $S \subset A$. Subsequently DAY proves

Remark 1. (i) Let $K$ be a compact, convex subset of a topological vector space over which its conjugate space is total. Then $K$ has at least one extreme point.

(ii) The set $\mathfrak{A}$ of all extremal sets $A$ of $K$ has a minimal element which is a singleton consisting of an extreme point. By our dimension hypothesis remark 1 applies to $B$ (and hence to $B^{\prime}$ ); consequently, $K \subset B$ has extreme points.

Definition 3. For every $k \leqq K, C(k)$ denotes the smallest extremal set containing $k$. 
Obviously, the operator $C$ has the properties of extensionality, isotony and idempotence. If $k=\{V\}$, we write briefly $C(V)$ (as mentioned in section I).

Of particular interest for us are the internal points of a convex set and the facets [14]:

Definition 4. In a real vector space let $A$ be any convex set and $M(A)$ the linear manifold generated by $A$.

(i) $x \in A$ is called an internal point of $A$ relative to $M(A)$ iff for each line $g \leqq M(A)$ through $x$ there exists an open segment $S \leqq g \cap A$ with $x \in S$.

(ii) The set of all internal points of $A$ is abbreviated by $A^{i}$.

Thus the internal points are special passing points.

Definition 5. Let $K \neq \emptyset$ be any convex set in a real vector space. For each $x \in K$ we define the facet $A(x)$ in $K$ by

(i) $x \in A(x)$

(ii) for all $y \neq x: y \in A(x)$ iff $y \in K$ and the line $g(x, y)$ through $x$ and $y$ contains an open segment $S \leqq K$ with $x \in S$.

Henceforth, for any two elements $x_{1}, x_{2}$ of a real vector space $] x_{1}, x_{2}[$ and $\left[x_{1}, x_{2}\right]$ denote the open and closed (line) segments, respectively. From the definitions 1,4 and 4 we infer

Consequence 1. The extreme points of $K$ are those points whose facets in $K$ are singletons.

Consequence 2. The internal points of $K$ are those points whose facets in $K$ are equal to $K$.

BOURBAKI [14] p. 152 has suggested the following statements now proved for the sake of completeness of this exposition:

Remark 2. There holds $[x, y] \subset A(x)$ for every $y \neq x$ with $y \in A(x)$.

Proof. Since there exists $] y_{1}, y[\subset K$ with $x \in] y_{1}, y\left[\right.$, any $y_{\lambda}=\lambda x$ $+(1-\lambda) y, \lambda \in] o, 1\left[\subset \boldsymbol{R}_{+}\right.$defines the open segment $] y_{1}, y_{\lambda}[\subset K$ because $K$ is convex. Besides, there holds $x \in] y_{1}, y_{\lambda}\left[\right.$, thus $y_{\lambda} \in A(x)$. -

Lemma 1. There holds with the hypothesis of definition 5: for each $x \in K$, the facet $A(x)$ is the largest convex set $A \subset K$ for $x$ to be an internal point of $A$ relative to $M(A)$.

Proof. To prove the convexity of $A(x)$, distinguish two cases:

1) Given $y_{1}, y_{2}, x \in A(x)$ with $y_{1} \neq y_{2}$ so that $x \in g\left(y_{1}, y_{2}\right)$. Then by remark 2, any convex combination of $y_{1}$ and $y_{2}$ lies in $A(x)$.

2) Given $y_{1}, y_{2}, x \in A(x)$ with $y_{1} \neq y_{2}$ so that $x \notin g\left(y_{1}, y_{2}\right)$. Then there exists a unique plane $p\left(y_{1}, y_{2}, x\right)$ through $y_{1}, y_{2}, x$ in $M(A(x))$. According to remark 2 there exist $] \bar{y}_{1}, y_{1}[,] \bar{y}_{2}, y_{2}[$ so that $x \in] \bar{y}_{1}, y_{1}[$, $x \in] \bar{y}_{2}, y_{2}[,] \bar{y}_{1}, y_{1}\left[\subset K \cap p\left(y_{1}, y_{2}, x\right)\right.$ and $] \bar{y}_{2}, y_{2}\left[\subset K \cap p\left(y_{1}, y_{2}, x\right)\right.$. Since $y_{1} \neq y_{2}$, so $\bar{y}_{1} \neq \bar{y}_{2}$ too. Hence $] \bar{y}_{1}, \bar{y}_{2}[\neq \emptyset$ with $] \bar{y}_{1}, \bar{y}_{2}\left[\subset K \cap p\left(y_{1}, y_{2}, x\right)\right.$. Therefore, for any $y \in] y_{1}, y_{2}[$ we can find an element $\bar{y} \in] \bar{y}_{1}, \bar{y}_{2}$ [ so that $x \in] y, \bar{y}[$, hence $y$ (and $\bar{y}) \in A(x)$. 
To prove that $A(x)$ is the largest convex set $A \subset K$ with $x$ an internal point of $A$ relative to $M(A)$, we assume the existence of another convex set $A_{1}$ with $x \in A(x) \subset A_{1} \subset K$ such that $x$ is internal relative to $M\left(A_{1}\right)$. Then there exists $y \in A_{1} \backslash A(x) \subset K$ so that $\left.x \in\right] y, \bar{y}[$ with $\bar{y} \in K$. Hence $\bar{y} \in A(x)$ and therefore $y \in A(x)$ too, which is a contradiction. $-\downarrow$

Lemma 2. There holds with the hypothesis of definition 5:

(i) For each $y \in A(x): A(y)$ in $K$ equals the facet $A_{x}(y)$ of $y$ in $A(x)$.

(ii) $A(x)=A(y)$ iff $y \in A(x)^{i}$.

(iii) The inclusion $\leqq$ being a partial ordering in the set of all facets $A(x)$ in $K$ and in the set of all $M(A(x))$, there exists an order isomorphism between the two sets.

(iv) If the dimension $\operatorname{dim} A(x)$ is finite, then $A(y) \subset A(x)$ implies $\operatorname{dim} A(y)<\operatorname{dim} A(x)$.

Proof. (i) Since obviously $A_{x}(y) \leqq A(y)$, it suffices to prove $A(y) \leqq$ $\leqq A(x)$ for every $y \in A(x)$ : (i) is trivial if $x$ or $y \in A(x)$ are extreme points of $K$. This case excluded, regard any pair $y_{1}, y_{2} \in K$ with $y_{1} \neq y_{2}$, $y \in] y_{1}, y_{2}$ [ (i.e. $\left.y_{1}, y_{2} \in A(y)\right)$ and ] $y_{1}, y_{2}\left[\notin g(x, y)\right.$. Since $x \in A(x)^{i}$ there exists $x_{1} \in A(x)$ so that $\left.x \in\right] y, x_{1}\left[\right.$. Thus $g\left(y_{1}, y_{2}\right)$ and $g(x, y)$ lie in the (unique) plane $p\left(y_{1}, y_{2}, x\right)$. Therefore $g\left(y_{2}, x\right) \subset p\left(y_{1}, y_{2}, x_{1}\right)$ is valid and thus $\emptyset \neq] y, x_{1}\left[\cap g\left(y_{2}, x\right)=\left\{x_{2}\right\} \subset K\right.$. So we obtain $\left.x \in\right] y_{2}, x_{2}[\subset K$, hence $y_{2} \in A(x)$ (and also $y_{1} \in A(x)$ ).

(ii) 1) For all $y \in A(x)^{i}$ there holds $A(y) \leqq A(x)$ by (i); thus $A(y)$ $=A(x)$ by lemma 1 .

2) If $A(y)=A(x)$, then $A(y)^{i}=A(x)^{i}$ with $y \in A(x)^{i}$.

(iii) 1) For any $x, y \in K, M(A(y)) \subset M(A(x))$ implies $A(y) \subset A(x)$ by definition 4 (i).

2) $A(y) \subset A(x)$ implies $M(A(y)) \leqq M(A(x))$ and $y \in A(x) \backslash A(x)^{i}$ by (ii). Assume $M(A(y))=M(A(x))$. Since always $y \in A(y)^{i}$ (relative to $M(A(y)))$, so every line in $M(A(x))$ passing through $y$ contains $y$ in an open segment belonging to $A(x)$. Thus $y \in A(x)^{i}$, which is a contradiction.

(iv) By (iii) $A(y) \subset A(x)$ implies $M(A(y)) \subset M(A(x))$. Since $\operatorname{dim} A(x)$ : $=\operatorname{dim} M(A(x))$ is finite, $\operatorname{dim} M(A(y))<\operatorname{dim} M(A(x))$ is consequently valid; hence the assertion. -

Let us again focus our attention on $\left(B, B^{\prime}\right)$ where $K C B$ is closed and convex. $B$ being finite-dimensional, we shall prove that, with respect to the definitions 2,3 and 5, the extremal sets of $K$ are facets in $K$. To this end we prove first

Lemma 3. Every facet $A(V)$ in $K \subset B$ is closed.

Proof. 1) If $A(V)$ is a singleton, then the assertion is true because $B$ is a $T_{4}$-space.

2) Suppose $A(V) \neq\{V\}$ and $A(V) \subset K$. By lemma 2 (iii) we have $M(A(V)) \subset M(K)$ and, since $\operatorname{dim} B<\infty, M(A(V))$ is closed. Denoting by - the closure operation, we get the relation $A(V)^{-} \subset M(A(V))^{-}$ 
$=M(A(V))$. Let $V_{1} \in A(V)$ - be an accumulation point of $A(V)$. Since $V_{1} \in M(A(V))$ and $V \in A(V)^{i}$, there exists $V_{2} \in A(V)$ in $g\left(V_{1}, V\right)$ so that $V \in] V_{1}, V_{2}\left[\right.$, hence $V_{1} \in A(V)$ by definition 5 . - -

Theorem 2. The set $\mathfrak{A}$ of all extremal sets of $K$ is equal to the set $\mathfrak{A}_{f}$ of all facets in $K$.

Proof. To prove $\mathfrak{A}_{f} \leqq \mathfrak{A}$ we note that, according to definition 5 and the lemmas 1 and $3, A(V)$ is non-empty, convex and closed. Let us verify definition 2 (ii) for any $A(V) \in \mathfrak{A}_{f}$ : given any $] V_{1}, V_{2}[\subset K$ with $A(V) \cap] V_{1}, V_{2}\left[\neq \emptyset\right.$, then there holds for any $V_{3}$ in the intersection ]$V_{1}, V_{2}\left[\subset A\left(V_{3}\right) \leqq A(V)\right.$ by lemma $2(\mathrm{i})$. Hence $A(V) \in \mathcal{A}$.

To prove $\mathfrak{A} \leqq \mathfrak{A}_{f}$ observe that, because of the finite dimension of $B$, every $A \in \mathfrak{A}$ contains a simplex of the same dimension as $A$ has. The barycentre $V_{b}$ of this simplex is an internal point of $A$, i.e. $V_{b} \in A^{i}$. So by the definitions 2 and $5 A\left(V_{b}\right) \leqq A$. Conversely, for every $V \in A$ with $V \neq V_{b}$ there exists $\bar{V} \in A$ with $\left.V_{b} \in\right] V, \bar{V}\left[\right.$ since $V_{b} \in A^{i}$; thus $V \in A\left(V_{b}\right) .-$

Corollary 1. $C(V)=A(V)$ for all $V \in K$.

Proof. Since $A(V) \in \mathfrak{A}, C(V) \leqq A(V)$ is evident. Assume $C(V) \subset A(V)$. Then by theorem 2 there exists $A\left(V_{1}\right)$ with $C(V)=A\left(V_{1}\right)$. Since $V \in A\left(V_{1}\right)$, so $A(V) \leqq A\left(V_{1}\right)$ by lemma 2 (i), which is a contradiction. -

\section{Corollary 2. $\mathfrak{A}=\hat{W}$.}

Proof. By theorem 6 in [3] every $C(V) \in \mathfrak{A}$ can be written as $C(V)$ $=K_{o} \hat{L}_{o}(V)$. Theorem 2 completes the proof. -1

Corollary 3. $\mathfrak{A}$ is dually order-isomorphic to $\hat{U}$.

Proof. The assertion follows from corollary 2 and the dual order isomorphism between $\hat{U}$ and $\hat{W} .-$

Theorem 3. $C(k)=K_{o} \hat{L}_{o}(k)$ for every $k \leqq K$.

Proof. $C(k) \leqq K_{o} \hat{L}_{o}(k)$ is trivially valid. Since for each $V \in k$ $C(V) \leqq C(k)$, we can infer from theorem 6 in [3]:

$$
\left.K_{o} \hat{L}_{o}(k)=K_{o}\left(\bigcap_{V \in k} \hat{L}_{o}(V)\right)=\underset{V \in k}{\bigvee} K_{o} \hat{L}_{o}(V)=\underset{V \in k}{\bigvee} C(V) \leqq C(k) .\right\lrcorner
$$

Corollary 1. $C(k)=\underset{V \in k}{\vee} C(V)$ for every $k \leqq K$.

Corollary 2. For all finite $k \leqq K$.

$$
\left.C(k)=C\left(\sum_{V \in k} \lambda_{V} V\right) \text { with } \sum_{V \in k} \lambda_{V}=1, \lambda_{V} \in\right] o, 1[.
$$

Proof. Since $\hat{L}_{o}\left(\sum_{V \in k} \lambda_{V} V\right)=\bigcap_{V \in k} \hat{L}_{o}(V)$, the assertion results from corollary 1. $\lrcorner$

Remark 3. Evidently, if $V_{e}$ is an extreme point of $K$, then $V_{e}$ is also an extreme point of each $A \in \mathfrak{A}$ with $V_{e} \in A$. Conversely, if $V_{e}$ is an extreme point of any $A \in \mathfrak{A}$, then it is also an extreme point of $K$. This follows from the fact that for each $] V_{1}, V_{2}\left[\subset K\right.$ with $\left.V_{e} \in\right] V_{1}, V_{2}[$ ]$V_{1}, V_{2}[\subset A$ is also valid, which is contradictory. 
This enables us to point out a bijection between the set of all extreme points of $K$ and the set $A(G)$ of all atoms of $G$.

Theorem 4. Every atom of $W$ is a singleton.

Proof. By lemma 2 (iv) $W$ satisfies the descending chain condition, i.e. $W$ is atomic, thus $G$ too. Remark 1 guarantees that every element of $W$ has an extreme point. Therefore, if $P \in G$ is an atom of $G$, for an extreme point $V_{p} \in K_{1}(P) C\left(V_{p}\right)=\left\{V_{p}\right\}$ holds by remark 3. Since $W=\mathfrak{A}$ and $K_{1}(P)$ is an atom of $W$, so $K_{1}(P)=\left\{V_{p}\right\}$. -

Corollary. For all $E \in G$ :

$E=\bigvee_{i=1}^{n} P_{i}$ implies $K_{1}(E)=C\left(\sum_{i=1}^{n} \lambda_{i} V_{P_{i}}\right)$, where $\sum_{i=1}^{n} \lambda_{i}=1, o<\lambda_{i}<1$.

Proof. By theorem 4 we have $K_{1}(E)=\bigvee_{i=1}^{n} K_{1}\left(P_{i}\right)=\bigvee_{i=1}^{\mathrm{V}} C\left(V_{P_{i}}\right)$. Applying the corollaries 1 and 2 of theorem 3, we obtain the assertion. -

When we introduced the concept of a facet, there would have been another way of defining it, which we are going to make up for. The equivalence with definition 5 will be evident. Using the hypotheses in definition 5, we define for every $x \in A: y \in A(x)$ iff $y \in A$ and there exist $\bar{y} \in A$ and $\lambda \in] o, 1\left[\subset \boldsymbol{R}_{+}\right.$so that $x=\lambda y+(1-\lambda) \bar{y}$.

It is this definition that has a direct physically intuitive meaning, as demonstrated in $[4-6]$.

\section{The Duality of $\left(K_{1}(E), \hat{L}_{E}\right)$}

According to section I we have for all $E \in G$ :

$$
\begin{aligned}
K_{1}(E) & =K_{o}\left(E^{\prime}\right)=\left(K_{o}(E)\right)^{\prime} \in W=\hat{W} \text { and } \\
\hat{L}_{E}: & =\{F \mid F \in \hat{L}, F \leqq E\}=\hat{L}_{o} K_{o}(E) \in \hat{U} .
\end{aligned}
$$

The purpose of this section is to prove all those axiomatic properties for $\left(K_{1}(E), \hat{L}_{E}\right)$ which were postulated of $(K, \hat{L})$. In the sequel we shall use the abbreviations for all $E \in G$ :

$$
\begin{gathered}
F_{1} \underset{K_{1}(E)}{\overline{(}} F_{2} \text { iff } \mu\left(V, F_{1}\right)=\mu\left(V, F_{2}\right) \quad \text { for all } V \in K_{1}(E) \\
V_{1} \underset{\overline{\bar{L}_{E}}}{=} V_{2} \text { iff } \mu\left(V_{1}, F\right)=\mu\left(V_{2}, F\right) \quad \text { for all } F \in \hat{L}_{E} \text {, with } K_{1}(\mathbf{1})=K
\end{gathered}
$$

and $\hat{L}_{\mathbf{1}}=\hat{L}$ holding.

Furthermore, we introduce the notations

$$
\begin{array}{lll}
K_{o}^{E}(l):=K_{o}(l) \cap K_{1}(E) & \text { for all } & l \leqq \hat{L} \\
K_{1}^{E}(l):=K_{1}(l) \cap K_{1}(E) & \text { for all } & l \leqq \hat{L} \\
\hat{L}_{o}^{E}(k):=\hat{L}_{o}(k) \cap \hat{L}_{E} & \text { for all } & k \leqq K \\
\hat{L}_{1}^{E}(k):=\hat{L}_{1}(k) \cap \hat{L}_{E} & \text { for all } & k \leqq K .
\end{array}
$$


So we are able to verify the proposition of axiom 2 a for $\left(K_{1}(E), \hat{L}_{E}\right)$ in

Theorem 5. For every pair $F_{1}, F_{2} \in \hat{L}_{E}$ there exists $F_{3} \in \hat{L}_{E}$ so that $F_{1} \leqq F_{K_{1}(E)}, F_{2} \leqq F_{3}$ and $K_{o}^{E}\left(F_{3}\right) \supseteqq K_{o}^{E}\left(F_{1}\right) \cap K_{o}^{E}\left(F_{2}\right)$.

Proof. For every $F_{1}, F_{2} \in \hat{L}_{E}$ there exists, by axiom 2 a, $F_{3} \in \hat{L}$ so that $F_{1} \leqq F_{3}, F_{2} \leqq F_{3}$ and $K_{o}\left(F_{3}\right) \supseteqq K_{o}\left(F_{1}\right) \cap K_{o}\left(F_{2}\right)$. Since always $K_{o}\left(F_{3}\right) \leqq$ $\leqq K_{o}\left(F_{1}\right) \cap K_{o}\left(F_{2}\right)$, so $K_{o}\left(F_{3}\right)=K_{o}\left(F_{1}\right) \cap K_{o}\left(F_{2}\right)$. Then from $K_{o}(E) \leqq$ $\leqq K_{o}\left(F_{1}\right) \cap K_{o}\left(F_{2}\right)$ there follows $K_{o}(E) \leqq K_{o}\left(F_{3}\right)$, hence $F_{3} \in \hat{L}_{E}$. The rest of the assertion is trivial. -1 $\left(K_{1}(E), \hat{L}_{E}\right)$ satisfies axiom 3 :

Theorem 6. For every pair $V_{1}, V_{2} \in K_{1}(E): \hat{L}_{o}^{E}\left(V_{1}\right)=\hat{L}_{o}^{E}\left(V_{2}\right)$ implies $C_{E}\left(V_{1}\right)=C_{E}\left(V_{2}\right)$.

Proof. First let us observe that by definition 3. $C_{E}\left(V_{i}\right):=C\left(V_{i}\right) \cap$ $\cap K_{1}(E)=C\left(V_{i}\right)$ because $V_{i} \in K_{1}(E),(i=1,2)$. Since $\hat{L}_{o}\left(V_{i}\right)=\hat{L}_{o}\left(C\left(V_{i}\right)\right)$, we can write for $i=1,2: \hat{L}_{o}^{E}\left(V_{i}\right)=\hat{L}_{o}\left(V_{i}\right) \cap \hat{L}_{o} K_{o}(E)=\hat{L}_{o}\left(C\left(V_{i}\right)\right) \cap$ $\cap \hat{L}_{o} K_{o}(E)=\hat{L}_{o}\left(C\left(V_{i}\right) \vee K_{o}(E)\right)$. By hypothesis $\hat{L}_{o}\left(C\left(V_{1}\right) \vee K_{o}(E)\right)$ $=\hat{L}_{o}\left(C\left(V_{2}\right) \vee K_{o}(E)\right)$, hence $C\left(V_{1}\right) \vee K_{o}(E)=C\left(V_{2}\right) \vee K_{o}(E)$. By orthomodularity of $W, C\left(V_{i}\right) \leqq K_{1}(E)$ implies $C\left(V_{i}\right)=K_{1}(E) \wedge\left(C\left(V_{i}\right)\right.$ $\left.\vee K_{o}(E)\right)$ and so, by the preceding equality, $C\left(V_{1}\right)=C\left(V_{2}\right) .-$

Theorem 7. $(\alpha),(\delta)$ and $(\varepsilon)$ of axiom 1 are satisfied by $\left(K_{1}(E), \hat{L}_{E}\right)$.

Proof. The restriction $\mu_{E}$ of $\mu$ to $K_{1}(E) \times \hat{L}_{E}$ satisfies $(\alpha)$ obviously. Since $O \in \hat{L}_{E},(\delta)$ is evident and so is $(\varepsilon)$ because $\mu_{E}(V, E)=1$ for all $V \in K_{1}(E)$ $\left(K_{1}(E), \hat{L}_{E}\right)$ satisfies $(\beta)$ of axiom 1 :

Theorem 8. For all $V_{1}, V_{2} \in K_{1}(E): V_{1} \overline{\overline{\hat{\hat{L}}}} V_{2}$ implies $V_{1}=V_{2}$.

Proof. From $V_{1}=V_{2}$ there follows $\hat{L}_{o}^{E}\left(V_{1}\right)=\hat{L}_{o}^{E}\left(V_{2}\right)$, thus by theorem $6 C\left(V_{1}\right)=C\left(V_{2}\right)$. Assume $V_{1} \neq V_{2}$. According to lemma $1, V_{i}$ are internal points, thus the line $g\left(V_{1}, V_{2}\right)$ meets the boundary of $C\left(V_{i}\right)$ in $\bar{V}_{1}$ and $\bar{V}_{2}$, which also satisfy $\bar{V}_{1} \neq \bar{V}_{2}$. Since $V_{i} \notin C\left(\bar{V}_{i}\right)$ by lemma 2 , $C\left(\bar{V}_{1}\right) \neq C\left(\bar{V}_{2}\right)$ must be valid. Moreover, there holds

$$
\left.V_{1}=\lambda \bar{V}_{1}+(1-\lambda) V_{2}, \lambda \in\right] o, 1\left[; \quad V_{2}=\nu \bar{V}_{2}+(1-\nu) V_{1}, \nu \in\right] o, 1[
$$

and from this and the hypothesis we infer at once $\mu\left(\bar{V}_{1}, F\right)=\mu\left(\bar{V}_{2}, F\right)$ for all $F \in \hat{L}_{E}$, i.e. $\bar{V}_{1} \overline{\hat{\hat{L}}}_{\bar{V}_{2}} \bar{V}_{2}$. Thus, by the same conclusions as for $V_{1}$ and $V_{2}, C\left(\bar{V}_{1}\right)=C\left(\bar{V}_{2}\right) ;$ which yields a contradiction. -

Lemma 4. For every $F \in \hat{L}_{E}$ the mapping $\mu(\cdot, F)$ attains its supremum on $K_{1}(E)$.

Proof. Notice that $K$ is compact, i.e. $\mu(\cdot, F)$ attains its supremum on $K$. Assume the existence of $V_{o} \in K \backslash K_{1}(E)$ such that $\mu\left(V_{o}, F\right)$ $=\sup \{\mu(V, F) \mid V \in K\}$ and disregard the trivial case $F=O$. Then we may define $\lambda_{o}:=\mu\left(V_{o}, F\right)^{-1}$ and by theorem 1 (ii) we obtain $\lambda_{o} F \in \hat{L}$. $\mu\left(V_{o}, \lambda_{o} F\right)=1$ for $V_{o} \notin K_{1}(E)$ implies $\lambda_{o} F \underset{K}{E} E$. On the other side,

14 Commun. math. Phys., Vol. 9 
$K_{o}\left(\lambda_{o} F\right)=K_{o}(F) \supseteqq K_{o}(E)$ implies $\lambda_{o} F \underset{K}{\leqq}$, which gives a contradiction. -1

Corollary. For all $F \in \hat{L}_{E}$ and $\lambda \in \boldsymbol{R}_{+}$:

$$
\lambda F \underset{K_{1}(E)}{\leqq} E \text { implies } \lambda F \underset{K}{\leqq} E .
$$

Proof. By lemma $4, \mu(\cdot, F)$ attains its supremum for $V_{o} \in K_{1}(E)$ and so does $\mu(\cdot, \lambda F)$. Then the hypothesis guarantees $o \leqq \mu(V, \lambda F) \leqq 1$ for all $V \in K$. Hence, by theorem 1 (ii), $\lambda F \in \hat{L}$ and again, because of $K_{o}(\lambda F)=K_{o}(F) \supseteqq K_{o}(E), \lambda F \leqq E .-$ $\left(K_{1}(E), \hat{L}_{E}\right)$ satisfies $(\gamma)$ of axiom 1 :

Theorem 9. For all $\bar{F}_{o}, \bar{F}_{o} \in \hat{L}_{E}: \bar{F}_{{ }_{\boldsymbol{K}_{1}(E)}}=\overline{\bar{F}}_{o}$ implies $\overline{\boldsymbol{F}}_{o} \overline{\bar{K}}_{\bar{K}}$.

Proof. In theorem 9 of [3] Lubwig proves a so-called "spectral" representation for any $F_{o} \in \hat{\hat{L}}=\hat{L}$, which is obtained by recurrently solving the following system with respect to $F_{o}: \alpha_{i} F_{i}=\alpha_{i} E_{i}-F_{i-1}$, $i \in[1, n] \subset \boldsymbol{N}$, where $E_{i}$ is determined by $K_{o}\left(F_{i-1}\right)=K_{o}\left(E_{i}\right), \alpha_{i}$ : $=\sup \left\{\mu\left(V, F_{i-1}\right) \mid V \in K\right\}, F_{n}=O, E_{i}>E_{i+1}$ and $F_{i-1} \leqq E_{i}$.

From our hypothesis we deduce $K_{o}^{E}\left(\bar{F}_{o}\right)=K_{o}^{E}\left(\overline{\bar{F}}_{o}\right)$. Since $K_{o}(E) \leqq$ $\leqq K_{o}\left(\bar{F}_{o}\right)$ and $K_{o}(E) \leqq K_{o}\left(\overline{\bar{F}}_{o}\right)$, orthomodularity of $W$ gives $K_{o}\left(\bar{F}_{o}\right)$ $=K_{o}(E) \vee\left(K_{o}\left(\bar{F}_{o}\right) \cap K_{1}(E)\right)=K_{o}(E) \vee K_{o}^{E}\left(\bar{F}_{o}\right)$ and an analogous equation for $K_{o}\left(\bar{F}_{o}\right)$. Thus we have $K_{o}\left(\bar{F}_{o}\right)=K_{o}\left(\bar{F}_{o}\right)$ and so $K_{o}\left(\bar{E}_{1}\right)=K_{o}\left(\bar{E}_{1}\right)$ holds in the above-mentioned spectral representation for $\bar{F}_{o}$ and $\bar{F}_{o}$. Moreover, from lemma 4 and the hypothesis we can infer

$$
\sup \left\{\mu\left(V, \bar{F}_{o}\right) \mid V \in K\right\}=\sup \left\{\mu\left(V, \overline{\bar{F}}_{o}\right) \mid V \in K\right\} .
$$

Therefore, $\bar{F}_{o}$ and $\bar{F}_{o}$ have the same spectral representations. $-\downarrow$

The axioms 1 and 2 a holding for $\left(K_{1}(E), \hat{L}_{E}\right)$, it is possible to define decision effects with respect to $\left(K_{1}(E), \hat{L}_{E}\right)$ in the same way as for $(K, \hat{L})$ in section I. For this purpose let us first observe that, for any $l_{o}^{E} \leqq \hat{L}_{E}$, the greatest $l_{g}^{E} \leqq \hat{L}_{E}$ such that $K_{o}^{E}\left(l_{o}^{E}\right)=K_{o}^{E}\left(l_{g}^{E}\right)$ satisfies $l_{g}^{E}=\hat{L}_{o}^{E} K_{o}^{E}\left(l_{g}^{E}\right)$ analogous to section I. Therefore $\hat{O}_{E}=\left\{l_{g}^{E} \mid l_{g}^{E} \leqq \hat{L}_{E}\right\}$ forms a complete sublattice of $\hat{O}$. $l_{g}^{E}$ being directed by theorem 5 , the element $e_{l_{g}^{E}} \in l_{g}^{E}$ defined by $\mu_{E}\left(V, e_{l_{g}}\right):=\sup \left\{\mu_{E}(V, F) \mid F \in l_{g}^{E}\right\}$ for all $V \in K_{1}(E)$ is the greatest element of $l_{g}^{E}$. Denote the set of all $e_{l_{g}^{E}}$ by $G_{E}$, which is a complete lattice because $\hat{U}_{E}$ is so. Then we intend to prove that $G_{E}$ is isomorphic to the segment $G(O, E) \leqq G$. Moreover, we shall see that any element of $G_{E}$ is the restriction of an element of $G(O, E)$ to $K_{1}(E)$. Conversely, any element of $G_{E}$ can be uniquely extended to $K$ so that it operates there linearly.

Lemma 5. Every $E_{1} \in G(O, E)$ determines an element of $G_{E}$.

Proof. Since $E_{1} \leqq E$, so $\hat{L}_{o} K_{o}\left(E_{1}\right) \leqq \hat{L}_{o} K_{o}(E)=\hat{L}_{E}$. That is every $l_{o} \leqq \hat{L}$ such that $K_{o}\left(l_{o}\right)=K_{o} \hat{L}_{o} K_{o}\left(E_{1}\right)=K_{o}\left(E_{1}\right)$ is contained in $\hat{L}_{E}$, hence 
$l_{0}^{E}=l_{o}$. Thus $K_{o}\left(l_{o}\right)=K_{o}\left(E_{1}\right)$ implies $K_{o}\left(l_{o}\right) \cap K_{1}(E)=K_{o}\left(E_{1}\right) \cap K_{1}(E)$, i.e. $K_{o}^{E}\left(l_{o}^{E}\right)=K_{o}^{E}\left(E_{1}\right)$. Therefore $\hat{L}_{o} K_{o}\left(E_{1}\right)$ is also the greatest $l_{o}^{E}$ and thus $E_{1} \mid K_{1}(E)$ is an element of $G_{E} \cdot-$

Lemma 6. Every e $\in G_{E}$ determines an element of $G(O, E)$.

Proof. Consider $l_{g}^{E}$ determining e. $l_{o}^{E} \leqq \hat{L}_{E}=\hat{L}_{o} K_{o}(E)$ implies $K_{o}(E) \leqq K_{o}\left(l_{o}^{E}\right)$. From orthomodularity there follows $K_{o}\left(l_{o}^{E}\right)=K_{o}(E)$ $\vee\left(K_{o}\left(l_{o}^{E}\right) \cap K_{1}(E)\right)$ and $K_{o}\left(l_{g}^{E}\right)=K_{o}(E) \vee\left(K_{o}\left(l_{g}^{E}\right) \cap K_{1}(E)\right)$. By hypothesis, $K_{o}^{E}\left(l_{o}^{E}\right)=K_{o}^{E}\left(l_{g}^{E}\right)$, hence $K_{o}\left(l_{o}^{E}\right)=K_{o}\left(l_{g}^{E}\right)$, which obviously expresses that $e$ has a unique extension to $K$. In other words, every $e \in G_{E}$ can be considered as the restriction of an element $E_{1} \in G(O, E)$ to $\left.K_{1}(E).\right\lrcorner$

Lemma 7. For all $F_{1}, F_{2} \in \hat{L}_{E}: F_{1}<F_{2}$ implies $F_{1} \underset{K_{1}(E)}{<} F_{2}$.

Proof. By theorem 1, $F_{2}-F_{1}=: \stackrel{K}{F} \in \hat{L}_{E}$. Assume ${\stackrel{K_{1}(E)}{F_{1}} \overline{K_{1}(E)}}_{F_{2}}$. Hence $F \underset{K_{1}(E)}{=} O$ and $K_{1}(E) \leqq K_{o}(F)$. Since also $K_{o}(E) \leqq K_{o}(F)$, we obtain $K_{1}(E) \vee K_{o}(E)=K \leqq K_{o}(F)$. Thus $F=\overline{\bar{K}} O$, which is a contradiction. -

Theorem 10. The lattices $G_{E}$ and $G(O, E)$ are orthoisomorphic.

Proof. The mapping $G(O, E) \rightarrow G_{E}$ defined by $E_{1} \rightarrow E_{1} \mid K_{1}(E)$ is bijective by the lemmas 5 and 6 . Lemma 7 says that it preserves order in both directions. The compatibility with the orthocomplementation is trivial. - -

Thus we are permitted in the sequal to identify $G_{E}$ and $G(O, E)$. We shall make use of it when proving axiom $2 \mathrm{~b}$ for $\left(K_{1}(E), \hat{L}_{E}\right)$. Before, however, we need a subsidiary proposition: defining $\left\{Y \mid Y \in B^{\prime}, Y\right.$ $=\lambda F, \lambda \in \boldsymbol{R}_{+}, F \in \hat{L}_{E}, o \leqq \mu(V, Y) \leqq 1$ for all $\left.V \in K_{1}(E)\right\}$ and $\widehat{\hat{L}}_{E}$ as its (weak) closure, we show

Lemma 8. $Y \in \widehat{\hat{L}}_{E}$ implies $Y \leqq E$.

Proof. Let $Y$ be an accumulation point of $\hat{L}_{E}$. Take any sequence $\left(Y_{i}\right)$ in the above-defined set which converges to $Y \in B^{\prime}$. Then by the corollary to lemma $4 Y_{i} \leqq E$, thus $Y \leqq E$. -

Theorem 11. For all $Y \in \widehat{\hat{L}}_{E}$ and $E_{1} \leqq E$ :

$$
K_{o}^{E}(Y) \supseteqq K_{o}^{E}\left(E_{1}\right) \text { implies } Y \underset{K_{1}(E)}{\leqq} E_{1} \text {. }
$$

Proof. By lemma 8 there holds $Y \leqq E$. By orthomodularity we have $K_{o}(Y)=K_{o}(E) \vee\left(K_{o}(Y) \cap K_{1}(E)\right), K_{o}\left(E_{1}\right)=K_{o}(E) \vee\left(K_{o}\left(E_{1}\right) \cap K_{1}(E)\right)$. Applying the hypothesis we obtain $K_{o}\left(E_{1}\right) \leqq K_{o}(Y)$. Thus axiom $2 \mathrm{~b}$ yields $Y \leqq E_{1} \cdot-$

Summarizing, we can conclude that statements and theorems valid for $(K, \hat{L})$ hold for $\left(K_{1}(E), \hat{L}_{E}\right)$ too. We shall particularly use theorem 1 with $\left(K_{1}(E), \hat{L}_{E}\right)$ substituted for $(K, \hat{L})$. 
The next definition includes some sets useful in the sequel.

Definition 6. For every $E \in G$ we denote:

(i) the (closed) linear hull of $K_{1}(E)$ by $B(E)$;

(ii) the (closed) linear hull of $\hat{L}_{E}$ by $B^{\prime}(E)$, and define;

(iii) $\mathscr{Q}(E):=\left\{X \mid X \in B(E), \mu_{E}(X, Y) \geqq o\right.$ for all $\left.Y \in \mathscr{P}(E)\right\}$;

(iv) $\mathscr{P}(E):=\left\{Y \mid Y \in B(E)^{\prime}, Y \underset{K_{1}(E)}{\geqq} O\right\}$ where $B(E)^{\prime}$ denotes the dual space of $B(E)$.

Thus, the following theorem is the most important conclusion drawn from the proved theorems in the whole.

Theorem 12. $B(E)^{\prime}$ is canonically isomorphic to $B^{\prime}(E)$. Hence we may identify $B(E)^{\prime}$ with $B^{\prime}(E)$ and, therefore, $\mathscr{Q}(E)$ and $\mathscr{P}(E)$ are the positive cones of $B(E)$ and $B^{\prime}(E)$, respectively. That is $\mathscr{Q}(E)=\underset{\lambda \in \boldsymbol{R}_{+}}{\bigcup} \lambda K_{1}(E)$ and $\mathscr{P}(E)=\bigcup_{\lambda \in \boldsymbol{R}_{+}} \lambda \hat{L}_{E}$.

Theorem 12 and its consequences create the facts upon which we shall base the construction of the subalgebra of $\mathscr{B}\left(B^{\prime}\right)$ as announced in section I.

\section{The Construction of the Algebra}

Section III shows that $\left(B(E), B^{\prime}(E)\right)$ is the pair of dual spaces pertaining to $\left(K_{1}(E), \hat{L}_{E}\right)$.

Definition 7. The projector mapping $B^{\prime}$ onto $B^{\prime}(E)$ is denoted by $\boldsymbol{T}_{E}$. Thus there holds $\operatorname{Im} \boldsymbol{T}_{E}=B^{\prime}(E)$.

Theorem 13. $\boldsymbol{T}_{E}$ is uniquely determined by $\boldsymbol{T}_{E} Y=Y \mid B(E)$ for all $Y \in B^{\prime}$, i.e. by $\mu\left(V, \boldsymbol{T}_{E} Y\right)=\mu(V, Y)$ for all $V \in K_{1}(E)$.

Proof. Consider the restriction of any $Y \in B^{\prime}$ to $B(E)$ defined by $\mu(X, Y \mid B(E))=\mu(X, Y)$ for all $X \in B(E)$. It follows that

1) $Y \mid B(E)$ is linear on $B(E)$.

2) $Y \mid B(E)$ is trivially unique as a restriction.

Therefore, by 1) and 2) an operator of $B^{\prime}$ onto $B^{\prime}(E)$ can be defined by $Y \rightarrow Y \mid B(E)$ for all $Y \in B^{\prime}$. Because of the bilinearity of $\mu$ this operator is linear. Restricted to $B^{\prime}(E)$ it obviously operates as the identity. Thus it is idempotent, i.e. it is equal to $\boldsymbol{T}_{E}$. Since $\mathscr{Q}(E)=\bigcup_{\lambda \in \boldsymbol{R}_{+}} \lambda K_{\mathbf{1}}(E)$ is generating with respect to $B(E), \boldsymbol{T}_{E}$ is completely determined by " $\mu\left(V, \boldsymbol{T}_{E} Y\right)=\mu(V, Y)$ for all $V \in K_{1}(E)$ and any $Y \in B^{\prime} " .-\triangle$

Corollary 1. (i) $\boldsymbol{T}_{E} F=F$ for all $F \in \hat{L}_{E}$, hence $\boldsymbol{T}_{E}\left[\hat{L}_{E}\right]=\hat{L}_{E}$ $=\hat{L}_{o} K_{o}(E) ; \quad$ (ii) $\boldsymbol{T}_{E}\left[\hat{L}_{1} K_{1}(E)\right]=\{E\} ; \quad$ (iii) $\boldsymbol{T}_{E}\left(\hat{L}_{o} K_{o}\left(E^{\prime}\right)\right]=\{O\}$ i.e. $B^{\prime}\left(E^{\prime}\right) \leqq \operatorname{Ker} \boldsymbol{T}_{E}$.

Proof. (i) Is evident. (ii) Results from $\hat{L}_{0} K_{o}(E) \cap \hat{L}_{1} K_{1}(E)=\{E\}$. (iii) Results from $K_{1}(E)=K_{o}\left(E^{\prime}\right) \leqq K_{o}(F)$ for all $F \leqq E^{\prime}$. $\downarrow$

Since always $B^{\prime}=\operatorname{Im} \boldsymbol{T}_{E} \oplus \operatorname{Ker} \boldsymbol{T}_{E}$ there arises the question when $B^{\prime}=B^{\prime}(E) \oplus B^{\prime}\left(E^{\prime}\right)$ holds, i.e. when $B^{\prime}\left(E^{\prime}\right)=\operatorname{Ker} \boldsymbol{T}_{E}$. The answer will be given in theorem 18 . 
Corollary 2. For all $E \in G: \boldsymbol{T}_{E}[\mathscr{P}]=\mathscr{P}(E)$.

Proof. By definition of $\boldsymbol{T}_{E}, \boldsymbol{T}_{E}[\hat{L}] \leqq \hat{L}_{E}$ and by (i) of corollary 1 $\hat{L}_{E} \leqq \boldsymbol{T}_{E}[\hat{L}]$. Thus $\boldsymbol{T}_{E}[\hat{L}]=\hat{L}_{E}$ and hence the assertion. -

Let us remember that $\mathscr{B}\left(B^{\prime}\right)$ denotes the $\boldsymbol{R}$-algebra of all linear operators over $B^{\prime}$. Then the set

$$
\mathscr{T}:=\left\{\boldsymbol{T} \mid \boldsymbol{T} \in \mathscr{B}\left(B^{\prime}\right), \boldsymbol{T}: \mathscr{P} \rightarrow \mathscr{P}\right\}
$$

forms a cone the elements of which are called positive. Since $\mathscr{P}$ satisfies $\mathscr{P} \cap(-\mathscr{P})=\{O\}, \mathscr{T}$ canonically induces a partial order in $\mathscr{B}\left(B^{\prime}\right)$ by "for all $\boldsymbol{H}_{1}, \boldsymbol{H}_{2} \in \mathscr{B}\left(B^{\prime}\right): \boldsymbol{H}_{1} \leqq \boldsymbol{H}_{2}$ iff $\boldsymbol{H}_{2}-\boldsymbol{H}_{1} \in \mathscr{T}$ " .

This is obviously equivalent to

$$
\text { " } \boldsymbol{H}_{\mathbf{1}} \leqq \boldsymbol{H}_{\mathbf{2}} \text { iff }\left(\boldsymbol{H}_{\mathbf{2}}-\boldsymbol{H}_{1}\right) Y \in \mathscr{P} \text { for all } Y \in \mathscr{P} \text { " . }
$$

By corollary 2 to theorem $13 \boldsymbol{T}_{E}$ is positive, hence we can prove

Theorem 14. $\boldsymbol{T}_{E}$ is isotone for all $E \in G$.

Proof. For all $Y_{1}, Y_{2} \in B^{\prime}$ with $Y_{2} \geqq Y_{1}$ there holds $Y_{2}-Y_{1} \in \mathscr{P}$. Hence $\boldsymbol{T}_{E}\left(Y_{2}-Y_{1}\right)=\boldsymbol{T}_{E} Y_{2}-\boldsymbol{T}_{E} Y_{1} \in \mathscr{P}(E) .-$

Theorem 15. For all $E_{1}, E_{2} \in G$ :

(i) $\boldsymbol{T}_{E_{2}} \boldsymbol{T}_{E_{1}}=\boldsymbol{T}_{E_{1}}$ iff $B^{\prime}\left(E_{1}\right) \leqq B^{\prime}\left(E_{2}\right)$

(ii) $B^{\prime}\left(E_{1}\right) \leqq B^{\prime}\left(E_{2}\right)$ iff $E_{1} \leqq E_{2}$

(iii) $E_{1} \leqq E_{2}$ iff $\boldsymbol{T}_{E_{1}} \boldsymbol{T}_{E_{2}}=\boldsymbol{T}_{E_{1}}$.

Proof. (i) is clear because $\boldsymbol{T}_{E_{i}}$ are projectors with $\operatorname{Im} \boldsymbol{T}_{E_{i}}=B^{\prime}\left(E_{i}\right)$ $(i=1,2)$.

(ii) By definition 6 (ii) $B^{\prime}\left(E_{1}\right) \leqq B^{\prime}\left(E_{2}\right)$ implies $\hat{L}_{E_{1}} \leqq \hat{L}_{E_{2}}$, thus $E_{1} \leqq E_{2}$. The reverse direction is also obvious.

(iii) Suppose $E_{1} \leqq E_{2}$. Because of theorem 1 it suffices to verify $\boldsymbol{T}_{E_{1}} \boldsymbol{T}_{E_{2}} F=\boldsymbol{T}_{E_{1}} F$ for all $F \in \hat{L}$. By theorem 13 there holds for all $F \in \hat{L}$ $\mu\left(V, \boldsymbol{T}_{E_{1}} \boldsymbol{T}_{E_{2}} F\right)=\mu\left(V, \boldsymbol{T}_{E_{2}} F\right)$ for all $V \in K_{1}\left(E_{1}\right)$; besides, $\mu\left(V, \boldsymbol{T}_{E_{2}} F\right)$ $=\mu(V, F)$ for all $V \in K_{1}\left(E_{2}\right)$. Since $K_{1}\left(E_{1}\right) \leqq K_{1}\left(E_{2}\right)$ by hypothesis, so there also follows $\mu\left(V, \boldsymbol{T}_{E_{1}} \boldsymbol{T}_{E_{2}} F\right)=\mu(V, F)$ for all $V \in K_{1}\left(E_{1}\right)$, i.e. $\boldsymbol{T}_{E_{1}} \boldsymbol{T}_{E_{2}} F=\boldsymbol{T}_{E_{1}} F$ for all $F \in \hat{L}$.

Conversely, suppose $\boldsymbol{T}_{E_{1}} \boldsymbol{T}_{E_{\mathbf{2}}}=\boldsymbol{T}_{E_{1}}$. By corollary 1 to theorem 13 we have $\boldsymbol{T}_{E_{1}} \boldsymbol{T}_{E_{2}} E_{1}=\boldsymbol{T}_{E_{1}} E_{1}=E_{1}$, i.e. $\mu\left(V, \boldsymbol{T}_{E_{1}} \boldsymbol{T}_{E_{2}} E_{1}\right)=\mu\left(V, \boldsymbol{T}_{E_{2}} E_{1}\right)$ $=\mu\left(V, E_{1}\right)=1$ for all $V \in K_{1}\left(E_{1}\right)$. On the other side, applying theorem 13 to $\boldsymbol{T}_{E_{3}}$, we obtain $\mu\left(V, \boldsymbol{T}_{E_{2}} E_{1}\right)=\mu\left(V, E_{1}\right)$ for all $V \in K_{1}\left(E_{2}\right)$. Altogether it consequently follows that $K_{1}\left(E_{1}\right) \leqq K_{1}\left(E_{2}\right)$, hence the assertion. - -

$\mathscr{T}(G):=\left\{\boldsymbol{T}_{E} \mid \boldsymbol{T}_{E} \in \mathscr{T}, E \in G\right\}$ being a projector set, there exists the usual partial order in $\mathscr{T}(G)$, which is defined by

$$
\boldsymbol{T}_{E_{1}} \underset{\mathscr{T}(G)}{\leqq} \boldsymbol{T}_{E_{2}} \text { iff } \boldsymbol{T}_{E_{2}} \boldsymbol{T}_{E_{1}}=\boldsymbol{T}_{E_{1}} .
$$

Notice that theorem 15 ensures the antisymmetry of this order relation. So we are able to formulate our main theorem: 
Theorem 16. The mapping $\Theta: G \rightarrow \mathscr{T}(G)$ defined by $\Theta(E)=\boldsymbol{T}_{E}$ for all $E \in G$ is a lattice ortho-isomorphism between $G$ and $\mathscr{T}(G)$.

Proof. Theorem 15 and the preceding definition of order in $\mathscr{T}(G)$ show immediately that $\Theta$ is an order isomorphism. Hence $\mathscr{T}(G)$ is a lattice with the notations

$$
\boldsymbol{T}_{E^{\prime}}=:\left(\boldsymbol{T}_{E}\right)^{\prime}, \quad \boldsymbol{T}_{E_{1} \wedge E_{2}}=: \boldsymbol{T}_{E_{1}} \wedge \boldsymbol{T}_{E_{2}}, \quad \boldsymbol{T}_{E_{1} \vee E_{2}}=: \boldsymbol{T}_{E_{1}} \vee \boldsymbol{T}_{E_{2}} . \downarrow
$$

Corollary. $\mathscr{T}(G)$ is an orthomodular lattice in $\mathscr{T} \subset \mathscr{B}\left(B^{\prime}\right)$.

Proof. $\boldsymbol{T}_{E_{1}} \underset{\boldsymbol{T}(G)}{\lessgtr} \boldsymbol{T}_{E_{2}}$ implies $E_{1} \leqq E_{2}$, hence the assertion. -

Theorem 17. For all $E_{1}, E_{2} \in G: \boldsymbol{T}_{E_{1}} \leqq \boldsymbol{T}_{E_{2}}$ implies $\boldsymbol{T}_{E_{1}} \lesseqgtr \boldsymbol{T}_{E_{2}}$.

Proof. $\boldsymbol{T}_{E_{1}} \leqq \boldsymbol{T}_{E_{2}}$ is equivalent to $\boldsymbol{T}_{E_{1}} Y \leqq \boldsymbol{T}_{E_{2}} Y$ for all $Y \in \mathscr{P}$. Thus $\boldsymbol{T}_{E_{1}} E_{1}=E_{1} \leqq \boldsymbol{T}_{E_{2}} E_{1} \leqq E_{2}$.

Remark 4. Concerning Hilbert-space, where $K$ is the set of all positive semidefinite Hermitean operators $V$ with $\operatorname{Tr}(V)=1$ and where $\hat{L}$ is the set of all Hermitean operators $F$ with $O \leqq F \leqq 1$ and $\mu(V, F)$ is given by $\operatorname{Tr}(V F)$, the operator $\boldsymbol{T}_{E}$ is determined by $\boldsymbol{T}_{E} F=E F E$ for all $F \in \hat{L}$ and any projector $E \in \hat{L}$. In this case we can easily verify that the converse implication in theorem 17 is also valid, but we failed to prove it generally.

Let us now settle the question, when $\operatorname{Ker} \boldsymbol{T}_{E}=\operatorname{Im} \boldsymbol{T}_{E^{\prime}}$ holds. As the model in remark 4 shows, the lattice isomorphism $\Theta$ between $G$ and $\mathscr{T}(G)$ is not always ortho-additive. The next theorem gives a necessary and sufficient condition for its ortho-additivity.

Theorem 18. "G Boolean" is equivalent to "T $\boldsymbol{T}_{E_{1} \backslash E_{2}}=\boldsymbol{T}_{E_{1}}+\boldsymbol{T}_{E_{3}}$ for all orthogonal $E_{1}, E_{2} \in G$ ".

Proof. If $G$ is Boolean, then each $E \in G$ is compatible with all $\bar{E} \in G$, i.e. $E=(E \wedge \bar{E}) \stackrel{\frac{1}{V}}{ }\left(E \wedge \bar{E}^{\prime}\right)=E \wedge \bar{E}+E \wedge \bar{E}^{\prime}$. By theorem 1 it suffices to calculate the action of $\boldsymbol{T}_{E_{1} \grave{V} E_{2}}$ on $\hat{L}$. Because any $F \in \hat{L}$ has the spectral representation (see [3], theorem 15) $F=\sum_{\nu=1}^{n} \lambda_{\nu} E^{\nu}$ with $\lambda_{\nu}>0$ and $E^{v}$ pairwise orthogonal, it even suffices to calculate $\boldsymbol{T}_{E_{1} \frac{1}{\vee} E_{2}} E^{v}$. By hypothesis $E^{\nu}=\left(E^{v} \wedge\left(E_{1} \stackrel{\frac{1}{\vee}}{\vee} E_{2}\right)\right) \frac{1}{\vee}\left(E^{v} \wedge\left(E_{1} \stackrel{\frac{1}{V}}{ } E_{2}\right)^{\prime}\right)$, and by corollary 1 to theorem $13 \boldsymbol{T}_{E_{1} \vee E_{2}}\left(E^{v} \wedge\left(E_{1} \frac{1}{\vee} E_{2}\right)^{\prime}\right)=O, \quad \boldsymbol{T}_{E_{1} \frac{1}{\vee} E_{2}}\left(E^{v} \wedge\left(E_{1} \stackrel{\perp}{\vee} E_{2}\right)\right)$ $=E^{v} \wedge\left(E_{1} \stackrel{\perp}{\vee} E_{2}\right)=\left(E^{v} \wedge E_{1}\right) \stackrel{\perp}{\vee}\left(E^{v} \wedge E_{2}\right)=E^{v} \wedge E_{1}+E^{v} \wedge E_{2}$. On the other side, $E^{v}=\left(E^{v} \wedge E_{i}\right) \stackrel{1}{\vee}\left(E^{v} \wedge E_{i}^{\prime}\right)$, hence $\boldsymbol{T}_{E_{i}} E^{v}=E^{v} \wedge E_{i}(i=1,2)$ by the same corollary. Thus the final result:

$$
\boldsymbol{T}_{E_{1} \grave{\vee} E_{2}} E^{v}=E^{v} \wedge E_{1}+E^{v} \wedge E_{2}=\boldsymbol{T}_{E_{1}} E^{v}+\boldsymbol{T}_{E_{2}} E^{v}
$$


Conversely, suppose $\boldsymbol{T}_{E_{1} \frac{1}{\vee} E_{2}}=\boldsymbol{T}_{E_{1}}+\boldsymbol{T}_{E_{3}}$ for all orthogonal $E_{1}, E_{2} \in G$. Especially, $\boldsymbol{T}_{E \frac{1}{V} E^{\prime}}=\boldsymbol{T}_{E}+\boldsymbol{T}_{E^{\prime}}=\mathbf{i d}_{B^{\prime}}$. Thus every $F \in \hat{L}$ is additively decomposed into two components such that $F=F_{1}+F_{\perp}$ with $F_{1} \leqq E$ and $F_{\perp} \leqq E^{\prime}$. In other words, any $F$ is reduced by all $E \in G$, that is any two elements of $G$ reduce each other. By theorem 42 in [2] two such elements of $G$ are compatible, i.e. $G$ is Boolean. -

As demonstrated in [2], it suffices to suppose $G$ to be irreducible, i.e. $Z(G)=\{O, 1\}, Z(G)$ denoting the centre of $G$. If $G$ is reducible, the structure of $\left(B, B^{\prime}\right)$ is, in fact, completely determined as soon as the structure of the irreducible components of $G$ is known. With $G$ reducible $B^{\prime}$ splits up into $B^{\prime}=\bigoplus_{\nu=1}^{n} B^{\prime}\left(E^{v}\right), n \leqq \operatorname{dim} B^{\prime}, E^{\nu}$ being (orthogonal) atoms of $Z(G)$. We will now concern ourselves with the converse. Remember that $A(G)$ denotes the set of all atoms of $G$.

Lemma 9. If $B^{\prime}=\bigoplus_{\nu=1}^{n}\left(B^{\prime}\right)_{v}, \quad n \leqq \operatorname{dim} B^{\prime}$, and $\bigcup_{\nu=1}^{n}\left(A(G) \cap\left(B^{\prime}\right)_{\nu}\right)$ $=A(G)$, then $\mathbf{1}=\underset{\nu=1}{\stackrel{\perp}{\bigvee}} E_{v}$, each $E_{\nu}$ being the greatest element of $G \cap\left(B^{\prime}\right)_{v}$.

Proof. 1) $A(G) \cap\left(B^{\prime}\right)_{\nu} \neq \emptyset$ for all $\nu \in[1, n] \subset \boldsymbol{N}$. For assume the existence of $v_{o} \in[1, n]$ such that $A(G) \cap\left(B^{\prime}\right)_{v_{0}}=\emptyset$. Since there exists a basis $b=\left\{P_{i} \mid P_{i} \in A(G), i \in\left[1, \operatorname{dim} B^{\prime}\right]\right\}$ of $B^{\prime}$, so $b \cap\left(B^{\prime}\right)_{v_{0}}=\emptyset$. Therefore, the linear hull of $b$, which is $B^{\prime}$, is contained in $\bigoplus_{\nu \neq \nu_{0}}\left(B^{\prime}\right)_{v}$,
which is a contradiction.

2) $G$ being segment-orthocomplemented, any $P_{i_{0}} \in b$ can be supplemented by $P_{\alpha_{\varrho}} \in A(G)$ such that $1=P_{i_{0}} \frac{1}{\mathrm{~V}}\left(\begin{array}{c}m \\ \stackrel{1}{V} \\ \varrho=1\end{array} P_{\alpha_{\ell}}\right)=P_{i_{0}}+\sum_{\varrho=1}^{m} P_{\alpha_{\ell}}$, $m<\operatorname{dim} B^{\prime}$. Then there holds for all $\nu \in[1, n]\left\{P_{i_{0}}, P_{\alpha_{1}}, \ldots, P_{\alpha_{m}}\right\}$ $\cap\left(B^{\prime}\right)_{v} \neq \emptyset$, for otherwise, there would be $v_{o}$ such that $\left\{P_{i_{0}}, P_{\alpha_{1}}, \ldots, P_{\alpha_{m}}\right\}$ $\cap\left(B^{\prime}\right)_{v_{0}}=\emptyset$ and so $\mathbf{1} \in \bigoplus_{\nu \neq v_{0}}\left(B^{\prime}\right)_{v}$. By 1$),\left(B^{\prime}\right)_{v_{0}}$ contains at least one $P_{v_{0}} \in A(G)$. Starting from $P_{v_{0}}$, we can again find $P_{\beta_{\varkappa}} \in A(G)$ such that $\mathbf{1}=P_{v_{0}} \frac{1}{\mathrm{~V}}\left(\begin{array}{c}\bar{m} \\ \underset{\gamma=1}{\frac{1}{V}} \\ P_{\beta_{x}}\end{array}\right)=P_{v_{0}}+\sum_{x=1}^{\bar{m}} P_{\beta_{x}}, \bar{m}<\operatorname{dim} B^{\prime}$. This, however, contradicts the fact that every element of $B^{\prime}$ has a unique representation by components of $\left(B^{\prime}\right)_{\nu}$ because $B^{\prime}$ is a direct sum.

In the above orthodecomposition of $\mathbf{1}$, let us collect all those atoms which belong to an arbitrary but fixed $\left(B^{\prime}\right)_{v}$. Their supremum (equal to their sum) is denoted by $E_{v}$. Thus we obtain

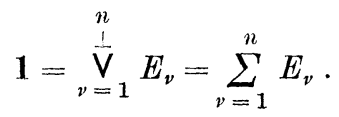


3) We prove that each $E_{\nu}$ is the greatest element of $G \cap\left(B^{\prime}\right)_{\nu}$. Starting from any atom in an arbitrary but fixed $\left(B^{\prime}\right)_{v}$, we can derive an orthodecomposition of $\mathbf{1}$ in the form indicated in 2). The supremum of all those atoms in this orthodecomposition which belong to the arbitrary but fixed $\left(B^{\prime}\right)_{\nu}$ must be equal to $E_{v}$ because of the uniqueness of such an orthodecomposition of $\mathbf{1}$. Thus all atoms belonging to this arbitrary but fixed $\left(B^{\prime}\right)_{v}$ are also atoms of $E_{v} \in\left(B^{\prime}\right)_{v}$ hence $E_{v}$ is the greatest element of $G \cap\left(B^{\prime}\right)_{v}$. -1

Corollary. Each $E_{v}$ of the orthodecomposition of 1 is compatible with all $E \in G$.

Proof. We have to prove $E_{v}=\left(E_{v} \wedge E\right) \stackrel{\frac{1}{\vee}}{\vee}\left(E_{v} \wedge E^{\prime}\right)$ for all $E \in G$. There obviously holds for all $E \in G\left(E \wedge E_{v}\right) \stackrel{\perp}{\vee}\left(E \wedge E_{v}^{\prime}\right) \leqq E$. Any $E$ can be decomposed by atoms of $G$. Since, by the hypothesis of lemma 9 ,

$\bigcup_{\nu=1}^{n}\left(A(G) \cap\left(B^{\prime}\right)_{\nu}\right)=A(G)$, the atoms of $E$ are also atoms of $E_{v}$ because the $E_{v}$ are the greatest elements in $G \cap\left(B^{\prime}\right)_{v}$. For an arbitrary but fixed $v_{o} \in[1, n]$ we deduce from lemma $9 E_{v_{o}}^{\prime}=1-E_{v_{o}}=\underset{\nu \neq v_{o}}{\frac{1}{V}} E_{\nu}$. Hence we conclude $E \leqq\left(E \wedge E_{v}\right) \stackrel{\perp}{\mathrm{V}}\left(E \wedge E_{v}\right)$. Thus $E=\left(E \wedge E_{v}\right) \stackrel{\perp}{\mathrm{V}}\left(E \wedge E_{v}^{\prime}\right)$ and by the orthomodularity of $G E_{\nu}=\left(E_{\nu} \wedge E\right) \stackrel{1}{\vee}\left(E_{\nu} \wedge E^{\prime}\right)$. $\lrcorner$

Theorem 19. With the hypotheses of lemma 9 and $\operatorname{dim} B^{\prime} \neq \operatorname{dim}\left(B^{\prime}\right)_{v}$ for all $v \in[1, n], G$ is reducible.

Proof. From the preceding corollary there follows $E_{v} \in Z(G)$ for all $E_{\nu}$ of the orthodecomposition of $\mathbf{1} \in G$. Since $B^{\prime}$ is supposed to be a nontrivial direct $\operatorname{sum}(n>1)$, there holds $E_{v} \neq O$ and $E_{v} \neq \mathbf{1}$ for all $v \in[1, n]$; hence the assertion. $-\longrightarrow$

Definition 8. (i) Let $\mathscr{A}(G)$ denote the (real) subalgebra of $\mathscr{B}\left(B^{\prime}\right)$ which is generated by $\mathscr{T}(G)$.

(ii) Let $\mathscr{A}(G)^{\prime}$ denote the commutant of $\mathscr{A}(G)$.

We will now establish a connexion between the operation of any subalgebra of $\mathscr{B}\left(B^{\prime}\right)$ and the reducibility of $G$. To this end let us first note the operation of $\boldsymbol{T}_{P}$ for any $P \in A(G)$ : by the proof of theorem 9 , any $F \in \hat{L}_{P}$ has the representation $F=\lambda P, \lambda \in[0,1]$. Hence $\boldsymbol{T}_{P} F=\lambda P$ $=\mu\left(V_{P}, F\right) P$ for every $F \in \hat{L}$, which can be deduced from theorem 13 and its corollary 2 .

Theorem 20. Let $\mathscr{A}$ be any subalgebra of $\mathscr{B}\left(B^{\prime}\right)$. If $\mathscr{A}$ is reducible, then so is $G$.

Proof. $\mathscr{A}$ reducible implies $\mathscr{A}^{\prime} \neq\left\{\alpha \mathbf{i d}_{B^{\prime}} \mid \mathbf{i d}_{B^{\prime}} \in \mathscr{B}\left(B^{\prime}\right), \alpha \in \boldsymbol{R}\right\}$. Consider any $\boldsymbol{A} \in \mathscr{A}^{\prime}$ such that $\boldsymbol{A} \neq \alpha \mathbf{i} \mathbf{i}_{B^{\prime}}$. $\boldsymbol{A}$ commutes especially with each $\boldsymbol{T}_{\boldsymbol{P}} \in \mathscr{B}\left(B^{\prime}\right): \boldsymbol{A T}_{P} P=\boldsymbol{A} \boldsymbol{P}=\boldsymbol{T}_{\boldsymbol{P}} \boldsymbol{A} P=\mu\left(V_{P}, \boldsymbol{A} P\right) \boldsymbol{P}=\alpha(P) P$ with $\alpha(P):=\mu\left(V_{P}, \boldsymbol{A} P\right)$, i.e. all $P \in A(G)$ are proper vectors of $\boldsymbol{A}$. 
Since $\boldsymbol{A} \neq \alpha \mathbf{i d}_{B^{\prime}}$, there exist at least two distinct proper values of $\boldsymbol{A}$. Suppose its total number to be $n\left(1<n \leqq \operatorname{dim} B^{\prime}\right)$ i.e. $\boldsymbol{A}$ decomposes $B^{\prime}$ into the non-trivial direct sum of the $n$ proper spaces $\left(B^{\prime}\right)_{\nu}$, each $\left(B^{\prime}\right)_{v}$ belonging to the proper value $\alpha_{v}$ of $\boldsymbol{A}$. Let $\left\{P_{i} \mid P_{i} \in A(G), i \in\left[1, \operatorname{dim} B^{\prime}\right]\right\}$ be a basis of $B^{\prime}$. Then [1, $\left.\operatorname{dim} B^{\prime}\right]$ is subdivided into $n$ disjoint sets $I_{v}$ such that $\alpha_{v}=\alpha\left(P_{i_{v}}\right)$ for all $i_{v} \in I_{v}$. Thus $\left\{P_{i_{v}} \mid i_{v} \in I_{v}\right\}$ is a basis of $\left(B^{\prime}\right)_{v}$. Any $P \in A(G)$ being a proper vector of $\boldsymbol{A}$, we have derived $\bigcup_{\nu=1}^{n}(A(G)$ $\left.\cap\left(B^{\prime}\right)_{v}\right)=A(G)$, i.e. the decisive hypothesis of theorem 19. As it is structurally not restrictive to require $G$ to be irreducible (see [2]), we can show the

Theorem 21. If $G$ is irreducible, then $\mathscr{A}=\mathscr{B}\left(B^{\prime}\right)$.

Proof. By theorem $20 \mathrm{~B}^{\prime}$ is a faithful and irreducible $\mathscr{A}$-module, hence $\mathscr{A}$ is primitive. Thus, by JACoBson's density theorem, $\mathscr{A}$ is algebraically dense in $\mathscr{B}\left(B^{\prime}\right)$ (see e.g. [19]) and so, because of the finite dimension of $B^{\prime}, \mathscr{A}=\mathscr{B}\left(B^{\prime}\right)$. In particular, $\mathscr{A}(G)=\mathscr{B}\left(B^{\prime}\right)$, which expresses that $\mathscr{A}(G)$ is a Baer-ring. $-\longrightarrow$

The concluding remarks of this paper are dedicated to the comparision of the projector $\boldsymbol{T}_{E}$ with the Sasaki-projection extensively used in $[7-11]$ and $[17,18]$ :

Definition 9. (i) If $G$ is orthomodular, a surjection $\Phi_{e}: G \rightarrow G(O, e)$ is defined by $\Phi_{e}(g)=e \wedge\left(g \vee e^{\prime}\right)$ for all $g \in G$ and any $e \in G([15])$.

(ii) Since $\Phi_{e}$ is idempotent, it is called Sasaki-projection.

As immediately to be verified, the following implications hold for $e, g \in G$ :

(1) $g \leqq e$ implies $\Phi_{e}(g)=g$ (by orthomodularity of $G$ )

(2) $e \leqq g$ implies $\Phi_{e}(g)=e$

(3) $g \leqq e^{\prime}$ implies $\Phi_{e}(g)=O$

(4) $e^{\prime} \leqq g$ implies $\Phi_{e}(g)=e \wedge g$.

According to NaKamura [16] there holds in an orthomodular $G$ with the definition " $e_{1} \mathscr{C} e_{2}$ iff $e_{1}=\left(e_{1} \wedge e_{2}\right) \stackrel{\frac{1}{V}}{ }\left(e_{1} \wedge e_{2}^{\prime}\right)$ ":

(5) $e_{1} \mathscr{C} e_{2}$ iff $\Phi_{e_{1}} \Phi_{e_{2}}=\Phi_{e_{2}} \Phi_{e_{1}}$

(6) $e_{1} \mathscr{C} e_{2}$ iff $e_{2} \mathscr{C} e_{1}$.

The model in remark 4 demonstrates that $\boldsymbol{T}_{E} \mid G$ is not generally a Sasaki-projection but if and only if $G$ is Boolean, which is a consequence of theorem 18. Whereas $\Phi_{e}$ is a v-homomorphism, $\boldsymbol{T}_{E} \mid G$ is so only in the Boolean case. The properties (1)-(4), however, are satisfied by $\boldsymbol{T}_{E}$ as can easily be seen by corollary 1 to theorem 13 and by the orthomodularity of $G$. We have, up to now, failed to settle the question whether $\boldsymbol{T}_{E}$ has property (5) or not.

Let us at last outline the main results of the co-ordinatizing procedure by Foults [7-11]: 
$\mathscr{S}(G)$ denotes that multiplicative semigroup of isotone mappings $\Phi$ of an orthomodular lattice $G$ into itself for which for each $\Phi \in \mathscr{S}(G)$ there exists one and only one $\Phi^{*} \in \mathscr{S}(G)$. Here, * is an involutory antiautomorphism on $\mathscr{S}(G) . \mathscr{S}(G)$ is a Baer-*-semigroup whose projection lattice $\mathscr{P}^{\prime} \mathscr{S}(G):=\left\{\Phi_{e} \mid e \in G, \Phi_{e} \in \mathscr{S}(G)\right\}$ is ortho-isomorphic to $G . \mathscr{S}(G)$ is then said to be a co-ordinate semigroup of $G$. Foulis has shown that $\mathscr{S}(G)$ is the smallest (with respect to homomorphisms) co-ordinate semigroup of $G$. Infimum, supremum and orthocomplementation in $\mathscr{P}^{\prime} \mathscr{S}(G)$ are determined merely by the *-mapping and the internal composition on $\mathscr{S}(G)$.

Thus there arise the following open questions in connexion with our exposition:

1) It is possible to express the lattice operations in $\mathscr{T}(G)$ by means of the internal compositions of $\mathscr{A}(G)$ ?

2) It is possible to define a *-mapping on $\mathscr{T}(G)$ only by order and orthocomplementation so that this involution is compatible with the internal compositions on $\mathscr{B}\left(B^{\prime}\right)$ and so that $\boldsymbol{T}_{E}=\boldsymbol{T}_{E} *$ ?

3) Suppose $\mathscr{B}\left(B^{\prime}\right)$ to have such an involution. Is $G$ then modular?

4) When does $\boldsymbol{T}_{E}$ satisfy " $E_{1} \mathscr{C} E_{2}$ iff $\boldsymbol{T}_{E_{1}} \boldsymbol{T}_{E_{2}}=\boldsymbol{T}_{E_{2}} \boldsymbol{T}_{E_{1}}$ "?

5) When does the converse of theorem 17 hold?

I thank Prof. G. LuDwia very much for suggesting this investigation and for his decisively stimulating criticism. I also thank Dr. K. Kraus, Dr. P. Stouz and Mr. W. Prediger for critical remarks.

\section{References}

1. Ludwig, G.: Versuch einer axiomatischen Grundlegung der Quantenmechanik und allgemeinerer physikalischer Theorien. Z. Physik 181, 233-260 (1964).

2. - Attempt of an axiomatic foundation of quantum mechanics and more general theories. II. Commun. Math. Phys. 4, 331-348 (1967).

3. - Attempt of an axiomatic foundation of quantum mechanics and more general theories. III. Commun. Math. Phys. 9, 1-12 (1968).

4. Hauptsätze über das Messen als Grundlage der Hilbert-Raumstruktur der Quantenmechanik. Z. Naturforsch. 22a, 1303-1323 (1967).

5. - Ein weiterer Hauptsatz über das Messen als Gundlage der Hilbert-Raumstruktur der Quantenmechanik. Z. Naturforsch. 22a, 1324-1327 (1967).

6. - English preprint combining [4] and [5].

7. Foulis, D. J.: Baer *-semigroups. Proc. Am. Math. Soc. 11, 648-654 (1960).

8. - Conditions for the modularity of an orthomodular lattice. Pacific J. Math. 11, 889-895 (1961).

9. - A note on orthomodular lattices. Portugal. Math. 21, 65-72 (1962).

10. - Relative inverses in Baer *-semigroups. Mich. Math. J. 10, 65-84 (1963).

11. - Semigroups co-ordinatizing orthomodular geometries. Can. J. Math. 17, $40-51(1965)$.

12. Mrues, P. E.: Order isomorphisms of $B^{*}$ algebras. Trans. Am. Math. Soc. 107, 217-236 (1963). 
13. Day, M. M.: Normed linear spaces. Second printing, N. F. Heft 21. BerlinGöttingen-Heidelberg: Springer 1962.

14. Bourbaki, N.: Espaces vectoriels topologiques, Chap. 1 et 2, deuxième édition. Paris: Hermann 1966.

15. SASAKI, U.: On orthocomplemented lattices satisfying the exchange axiom. J. Sci. Hiroshima Univ. Ser. A 17, 293-302 (1954).

16. NaKamura, M.: The permutability in a certain orthocomplemented lattice. Kodai Math. Sem. Rep. 9, 158-160 (1957).

17. Pool, J. C. T.: Baer *-semigroups and the logic of quantum mechanics. To be published in this journal.

18. - Semimodularity and the logic of quantum mechanics. Preprint March 1968.

19. Behrens, E. A.: Algebren, Bd. 97/97a. Mannheim: Bibliographisches Institut AG. 1965.

GÜNTER DäHN

Institut $f$. theor. Physik (1) d. Universität 355 Marburg (Lahn), Renthof 7 